\title{
Radiographic Changes vis-à-vis Tracheo-bronchial Aspirate Cytology Profile in Pneumonic Cattle
}

\author{
Asmita Narang, Charanjit Singh, Arun Anand, Swaran Singh Randhawa
}

10.18805/IJAR.B-4553

\begin{abstract}
Background: The study was conducted to establish the utility of radiography in the diagnosis of lung diseases in cattle and its correlation with different pneumonia diagnosed on the basis of TBA cytology.

Methods: Lateral chest radiography and tracheo-bronchial wash was performed in control $(n=21)$ and diseased group ( $n=55)$. Diseased group included cattle presented with respiratory signs and diagnosed with pulmonary diseases on the basis of history, physical and clinical examination and tracheo-bronchial wash cytology. Cytologic diagnosis was established as chronic pneumonia ( $n=24)$, acute pneumonia $(n=18)$, tuberculosis $(n=5)$ and aspiration pneumonia $(n=8)$. Survivability was also correlated with lung patterns in diseased cattle.

Result: Nodular interstitial pattern (27.3\%), unstructured interstitial pattern (25.4\%), bronchial pattern (20.0\%), pleural effusions $(12.7 \%)$, mixed lung patterns (10.9\%) and miliary interstitial pattern (4.54\%) was observed in diseased group. Unstructured interstitial pattern and pleural effusions were most evident in acute pneumonia. The radiographic findings in aspiration pneumonia did not correlate well with cytologic findings. Highest survivability was recorded in cows with bronchial pattern (81.8\%) and lowest in miliary interstitial pattern (zero per cent).
\end{abstract}

Key words: Cattle, Pneumonia, Radiography, Tracheo-bronchial aspirate.

\section{INTRODUCTION}

Respiratory disorders are next to gastrointestinal disorders in cattle. Cattle are more susceptible to respiratory diseases because of absence of inter-alveolar and inter-bronchiolar collateral ventilation, the high ventilation rate and strong resistance to airflow within the respiratory conducts (Lekeux, 1988). Antemortem diagnosis of lung diseases and their severity is relatively difficult. Under the present clinical setup, diagnosis of bovine respiratory diseases is based on clinical examination, hematology and radiography. Radiography can assist in the differentiation of atelectasis and consolidation, interstitial and exudative pneumonias, neoplasms, pleural effusions, pneumothorax and space-occupying lesions of the thorax.

There are many constraints to bovine chest radiography, the most important being accessibility to caudal lung lobes only. The other constraint is restriction to lateral radiographs as large amount of tissue prevents adequate exposure for ventrodorsal views. Moreover, radiographically detectable signs of lung disease can persist after clinical and clinicopathological recovery of the animal. This can mislead the diagnosis. Howsoever, it is imperative to conduct the radiography of thoraco-abdominal region as and when the clinical signs warrant its use for a pertinent diagnosis. Radiographically, the extent and severity of disease can be assessed. Corroboration of radiographic findings with clinical findings can not only help in the diagnosis of disorders but also helps in drawing the prognosis of the case.

Studies on radiography in pulmonary diseases of cattle are not well documented. Available literature lacks in the studies on the radiography of lung in cattle for the diagnosis
Department of Veterinary Medicine, Guru Angad Dev Veterinary and Animal Sciences University, Ludhiana-141 001, Punjab, India.

Corresponding Author: Asmita Narang, Department of Veterinary Medicine, Guru Angad Dev Veterinary and Animal Sciences University, Ludhiana-141 001, Punjab, India.

Email: asmi.vet@gmail.com

How to cite this article: Narang, A., Singh, C., Anand, A. and Randhawa, S.S. (2021). Radiographic Changes vis-à-vis Tracheobronchial Aspirate Cytology Profile in Pneumonic Cattle. Indian Journal of Animal Research. DOI: 10.18805/IJAR.B-4553.

Submitted: 02-06-2021 Accepted: 16-10-2021 Online: 19-11-2021

and prognosis of pneumonia. However, few studies had reported the correlation between radiographic signs and postmortem findings in cattle (Masseau et al. 2008). No previous study has reported the correlation between radiographic evaluation and tracheo-bronchial aspirate (TBA) cytology findings in cattle. Therefore, this study was planned to establish the utility of radiography in the detection of lung diseases in cattle and its correlation with different pneumonia diagnosed on the basis of TBA cytology.

\section{MATERIALS AND METHODS}

The study was carried out on adult crossbred cattle divided into two groups. Control group comprised of 21 cattle (Mean age: 5.57 years; range: 3-9 years) with no respiratory signs and normal TBA cytology. Diseased group included 55 cattle suspected for lung diseases on the basis of history and clinical manifestations (polypnoea, dyspnea, coughing). TBA 
was collected percutaneously in standing conscious animals using Large Animal Trans-Tracheal Wash Kit (MILA International, Inc. USA). Five $\mathrm{mL}$ aliquot of TBA was transferred to EDTA vials and centrifuged (2000 rpm, 5 minutes) to prepare and stain the sediment. The work was done during the period of August 2016 to July 2017, in Large Animal Clinics, Department of Veterinary Medicine, Guru Angad Dev Veterinary and Animal Sciences University, Ludhiana.

The cytological diagnosis was established as chronic pneumonia $(n=24)$, acute pneumonia $(n=18)$, aspiration pneumonia $(n=8)$ and tuberculosis $(n=5)$, supported by history and comprehensive clinical examination. Tuberculosis was further confirmed by cultural isolation and PCR. In chronic pneumonia, the diagnostic interpretation was based on highest proportion of activated macrophages in conjunction with rise in mean cell number (MCN) (cells/ HPF). In acute pneumonia, there was increase in MCN along with numerous degenerated neutrophils. Aspiration pneumonia was diagnosed on the basis of evidence of plant material in TBA smears along with history of drenching. In tuberculosis, MCN was higher with predominance of mononuclear cells primarily macrophages, lymphocytes, giant cells and fibroblasts. The radiographic findings were correlated with the cytologic findings in different pneumonia.

\section{Radiographic examination}

Lateral radiographs of chest region of all animals were taken, using kVp 90-115, mAS 50-70, FFD $90 \mathrm{~cm}$ by a $800 \mathrm{~mA}$ large animal X-ray machine (Siemens India, Mumbai). Radiographs were pro-cessed using computed radiography system (Kodak, India).

\section{Radiographic interpretation}

Radiographic findings in healthy and diseased animals were differentiated on the basis of radio-density and visibility of margins of blood vessels. The different lung patterns recorded were classified as mild, moderate and severe (Wilkins and Woolums, 2009).

\section{Interstitial lung pattern}

Characterization of the non-air-containing elements of the lungs including blood vessels and bronchi.

a) Normal:

b) Mild increase:

c) Moderate increase:

d) Marked increase:

\section{Nodular pattern}

Characterized by the presence of nodules; mild, moderate and marked increase was evaluated as mentioned above for interstitial pattern.

\section{Bronchial pattern}

Characterized by alterations in bronchial wall thickness and density, or in bronchial lumen diameter.

a) Normal: Bronchial structures seen in cross section appeared as small, thin-walled hollow rings between paired vessels.

b) Mild increase: The bronchial walls were barely distinguishable when viewed side-on and were not clearly visualized at the periphery of the lung field.

c) Moderate increase: A few thickened bronchial walls were evident in cross section ('doughnuts') at the periphery of the lung fields. Longitudinal sections appeared as tram lines reaching two-thirds of the way to the lung periphery

d) Marked increase: Extensive bronchial thickening was observed, extending far into the periphery of the visible lung field.

\section{Pleural effusions}

Apparent as distinct, horizontal fluid line; a soft tissue opacity that silhouettes the diaphragm present in the ventral thorax caused by pleural fluid.

\section{Mixed pattern}

More than one visible lung pattern.

\section{RESULTS AND DISCUSSION}

Limited studies are there on lung radiography in pneumonia in cattle. An attempt has been made to correlate TBA cytology with lung radiographic findings. Available studies had only described different pneumonias in cattle on the basis of histopathologic studies in dead cattle (Sorden et al. 2000, Elsiddig 2009, Gabinaitiene et al. 2011). Previously, isolated reports have appeared to correlate radiographic findings with histopathology or postmortem findings (Verschooten et al., 1974, Masseau et al., 2008). These studies showed that the radiographic findings did not always correlate with histopathologic findings and correlation was greatest in moderate to severe pulmonary lesions and mild changes might be overinterpreted.

Lung radiography in control group showed normal lungs with no characteristic lung pattern in 13 of 21 cattle, mild interstitial pattern in six and mild bronchial in two. Invariably, the margins of blood vessels were clear and well defined in the control group (Fig 1). Mild interstitial lung pattern in two healthy cows (aged 4 and 5 years) was not associated with pathologic changes in TBA cytology, so the findings were considered insignificant. Mild interstitial lung pattern in four healthy cows aged more than seven years was attributed to age-related changes as described in horses and cattle (8). Mild bronchial pattern in two healthy cows (aged 8 and 10 years) was ascribed to calcification of the bronchial cartilage with increasing age in older cows (Masseau et al. 2008).

In diseased group, several diffuse radiopaque areas were observed in visible lung parenchyma with indistict margins of blood vessels (Table 1; Fig 3 and 4). Increased radiopacity in lung parenchyma was a consistent finding in pneumonic cows. This has been earlier reported in cattle and buffaloes (Nykamp 2013, Tanwar 2016). The haze of 
increased pulmonary density reduced the details of contrast of the vascular markings and cardiac shadow. Highest proportion of cattle diagnosed for pneumonia showed interstitial nodular (27.3\%) (Fig 2), followed by unstructured interstitial (25.4\%) (Fig 3), bronchial pattern (20.0\%) (Fig 4), pleural effusions (12.7\%) (Fig 5), miliary interstitial (4.54\%) and mixed lung pattern (10.9\%) (Fig 6). There was no visible abnormality in 3.63 percent of diseased cattle. Masseau et al. (2008) reported 48 percent interstitial disease, 45 per cent cavitary lesions, 36 percent alveolar disease, 24 per

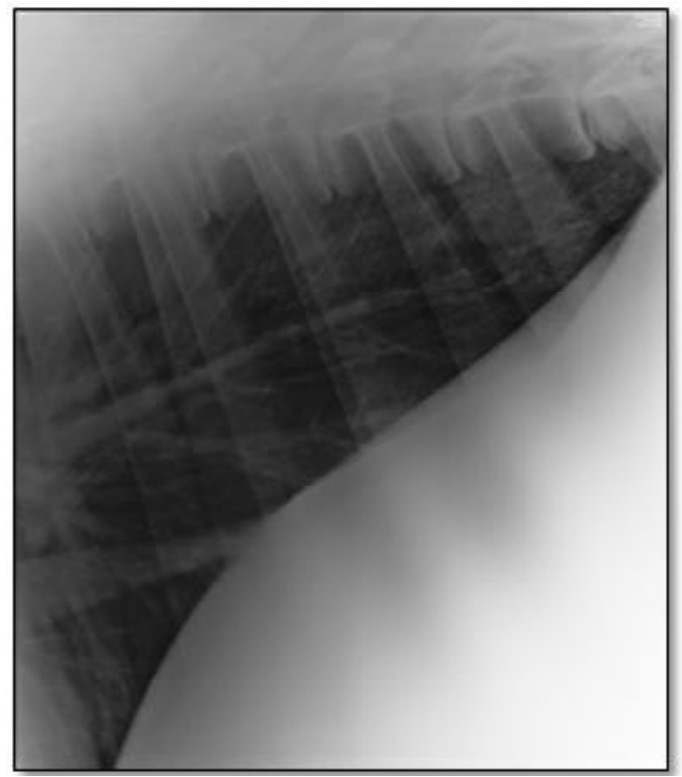

Fig 1: Caudolateral thoracic radiograph of a healthy cattle with distinct margins of blood vessels and normal opacity.

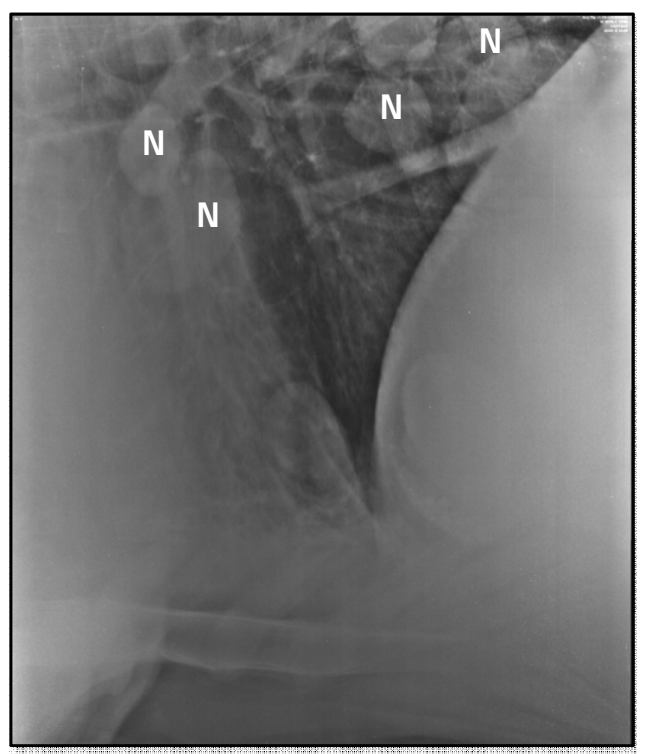

Fig 2: Caudolateral thoracic radiograph of cattle with increased radio-opacity; severe nodular pattern with multiple nodules $(N)$ in chronic pneumonia in cow. cent pneumomediastinum, 21 percent bronchial disease, and 19 per cent pleural effusions on thoracic radiography $(n=42)$. However, alveolar pattern was not observed in any cow in our study.

\section{Radiographic findings in different pneumonia \\ Chronic pneumonia}

Bronchial pattern was most common (37.5\%) in chronic pneumonia followed by nodular interstitial (29.2\%), unstructured interstitial (16.7\%) and miliary interstitial pattern

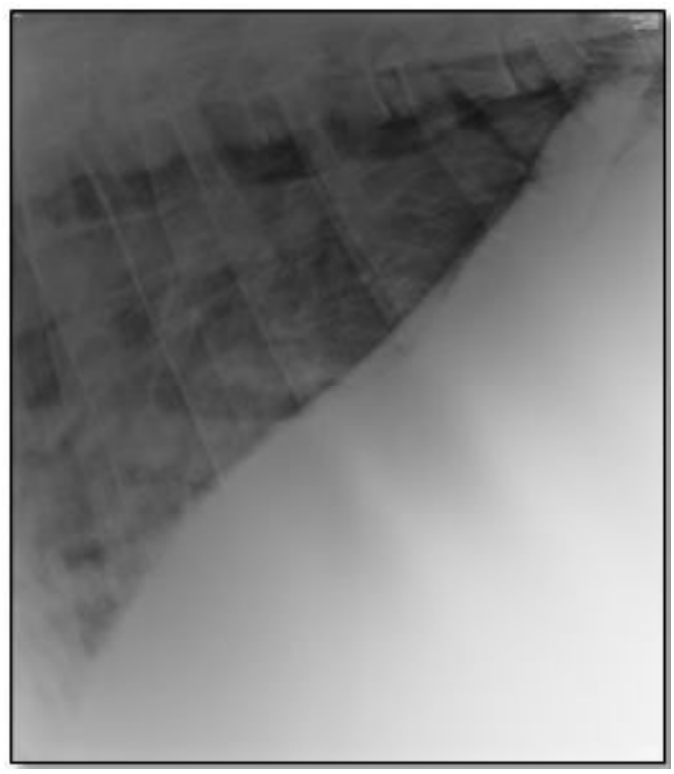

Fig 3: Caudolateral thoracic radiograph with increased opacity and indistinct margins of blood vessels, lesion is. consistent with an interstitial pattern in suppurative pneumonia in a cow

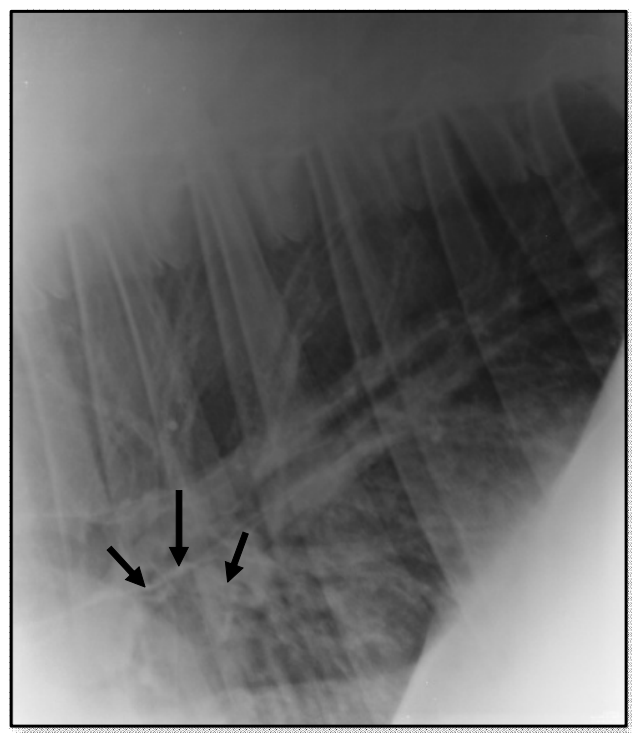

Fig 4: Caudolateral thoracic radiograph with increased opacity along with parallel lines and rings (doughnuts- solid arrow) of a cattle with bronchitis; lesion is consistent with bronchial pattern 
(4.16\%) (Table 1). Mixed lung patterns were evident in 12.5 percent $(3 / 24)$ cases of chronic pneumonia, in which two cows had bronchial and nodular interstitial pattern. Seventeen of 24 animals with chronic pneumonia survived (Table 1). Identifying cause of pulmonary nodules is difficult

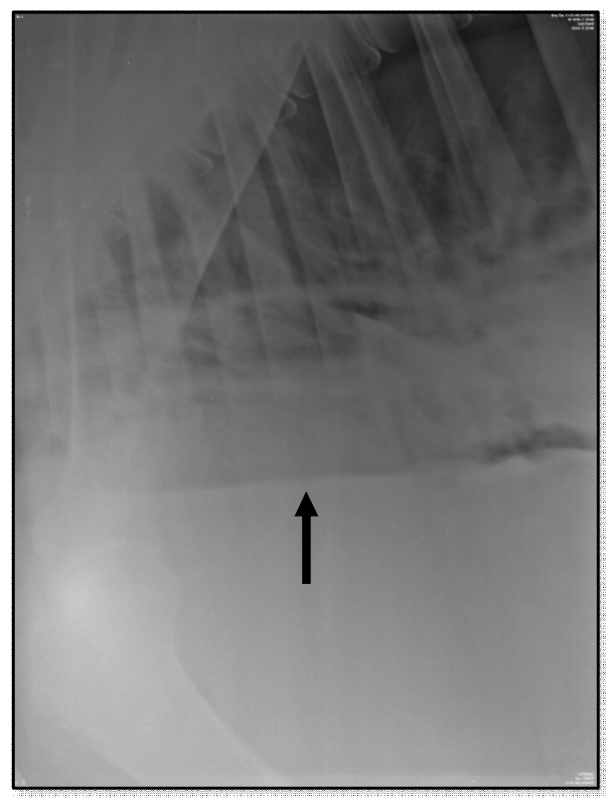

Fig 5: Caudolateral thoracic radiograph of a cattle with moderate pleural fluid; distinct, horizontal fluid line is evident (solid arrow), as there is considerable overlap in the radiographic appearances of tumors, granulomas and abscesses. Differential diagnoses of nodular interstitial pattern include noncavitary nodules, lung tumors, mycotic granuloma and abscessation. In the present study, neither the fungal

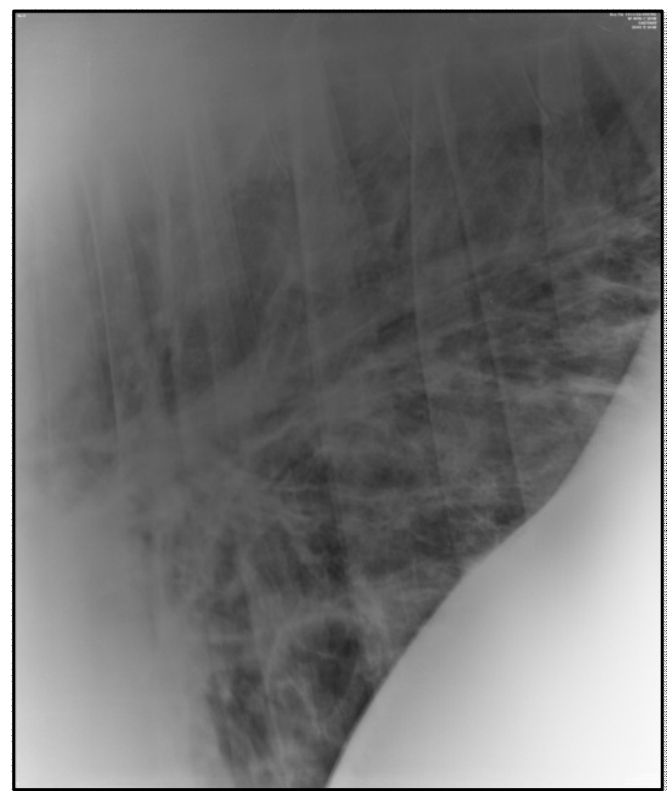

Fig 6: Caudolateral thoracic radiograph of a cow with severe interstitial-bronchial pattern; diffusely increased opacity in lungs, and multiple air bronchograms.

Table 1: Radiographic findings in diseased cattle

\begin{tabular}{|c|c|c|c|c|c|c|c|c|}
\hline $\begin{array}{c}\text { Lung patterns } \\
\text { Љ }\end{array}$ & $\begin{array}{c}\text { Lung affections } \\
\Rightarrow\end{array}$ & $\begin{array}{c}\text { Chronic } \\
\text { pneumonia } \\
(n=24)\end{array}$ & $\begin{array}{c}\text { Acute } \\
\text { pneumonia } \\
(\mathrm{n}=18)\end{array}$ & $\begin{array}{c}\text { Aspiration } \\
\text { pneumonia } \\
(n=8)\end{array}$ & $\begin{array}{c}\text { Tuberculosis } \\
\quad(n=5)\end{array}$ & TOTAL & $\begin{array}{l}\text { Proportion of } \\
\text { lung pattern } \\
(n=55)\end{array}$ & $\begin{array}{r}\text { Percent } \\
\text { surviv } \\
\text { ability }\end{array}$ \\
\hline Interstitial & Miliary & $1(0)$ & - & - & $2(0)$ & $3(0)$ & $5.45 \%$ & $0 \%$ \\
\hline \multirow[t]{2}{*}{ pattern } & Unstructured & $4(3)$ & $6(4)$ & $1(1)$ & - & $11(8)$ & $20.0 \%$ & $72.7 \%$ \\
\hline & Nodular & $7(4)$ & $1(1)$ & $4(3)$ & $3(0)$ & $15(8)$ & $27.3 \%$ & $53.3 \%$ \\
\hline Bronchial pattern & & $9(8)$ & $2(1)$ & - & - & $11(9)$ & $20 \%$ & $81.8 \%$ \\
\hline \multirow[t]{2}{*}{$\begin{array}{l}\text { Mixed } \\
\text { pattern }\end{array}$} & $\begin{array}{c}\text { Bronchial and unstructured } \\
\text { interstitial pattern }\end{array}$ & $1(0)$ & $2(1)$ & - & - & $3(1)$ & $5.45 \%$ & $33.3 \%$ \\
\hline & $\begin{array}{l}\text { Bronchial and nodular } \\
\text { interstitial pattern }\end{array}$ & $2(2)$ & $1(0)$ & - & - & $3(2)$ & $5.45 \%$ & $66.7 \%$ \\
\hline Pleural effusions & & - & $6(1)$ & $1(0)$ & - & $7(1)$ & $12.7 \%$ & $14.3 \%$ \\
\hline Normal lungs & & - & - & $2(1)$ & - & $2(1)$ & $3.63 \%$ & $50.0 \%$ \\
\hline
\end{tabular}

Values in parenthesis depict number of survived animals.

Table 2: Survivability in relation to severity of the radiographic changes in pulmonary affections.

\begin{tabular}{lccccc}
\hline Lung Pattern & Mild & Moderate & Marked & \multicolumn{2}{c}{ Total survived } \\
\hline Interstitial pattern & Miliary & - & - & $0(\mathbf{0} / 3)$ & $0 / 3$ \\
& Unstructured & $0(0 / 2)$ & $66.7(4 / 6)$ & $66.7(4 / 6)$ & $8 / 14$ \\
& Nodular & $75(3 / 4)$ & $75(3 / 4)$ & $28.6(2 / 7)$ & $8 / 15$ \\
Bronchial pattern & & $100(3 / 3)$ & $71.4(5 / 7)$ & $100(1 / 1)$ & $9 / 11$ \\
Mixed pattern & Bronchial and interstitial pattern & $100(1 / 1)$ & $0(0 / 2)$ & - & $1 / 3$ \\
& Bronchial and nodular pattern & - & $66.7(2 / 3)$ & - & $2 / 3$ \\
Pleural effusions & & $50(1 / 2)$ & $0(0 / 1)$ & $0(0 / 4)$ & $1 / 7$ \\
\hline
\end{tabular}

Values in parenthesis depict number of survived animals. 
elements, nor neoplastic cells were detected in TBA smears. Widespread spherical opacities were more likely to represent abscessation in horses (Nykamp 2013). In chronic pneumonia, bronchial pattern with thickening of bronchial wall and peribronchial tissues, was associated with chronic inflammation. However, bronchial pattern should always be interpreted with caution as reported bronchial pattern inconsequential in cattle considering it age related as in their study, the radiographic findings were not consistent with post mortem findings (Masseau et al., 2008). In contrast, present study showed diffused bronchial pattern on lung radiography

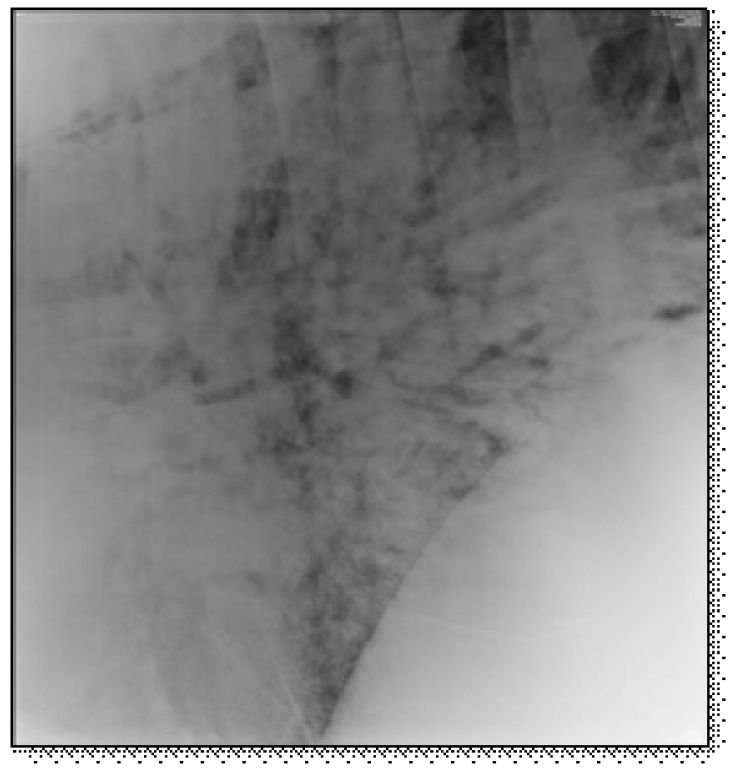

Fig 7: Caudolateral thoracic radiograph of cow with tuberculosis pneumonia; multiple nodules are present dorsocaudally; severe nodular interstitial pattern evident.

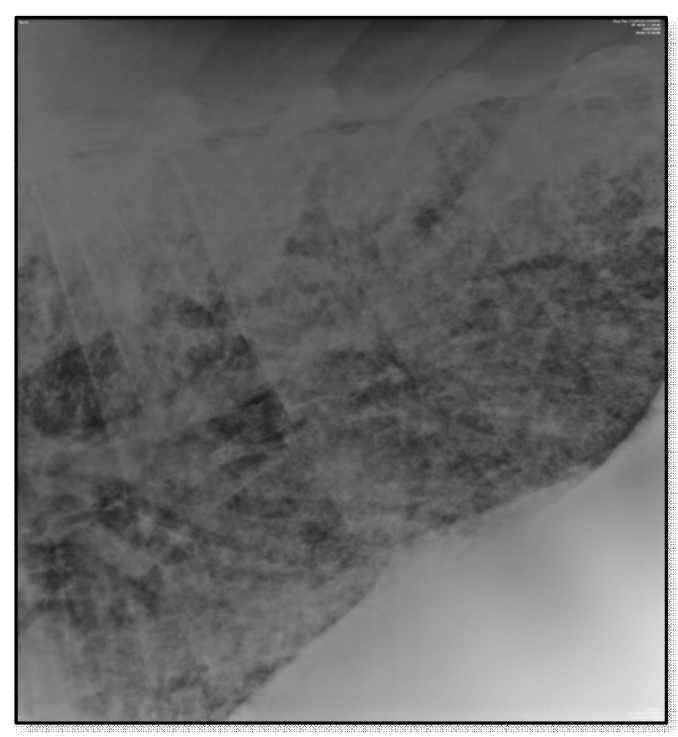

Fig 8: Caudolateral thoracic radiograph of cow with tuberculosis pneumonia; severe diffuse miliary interstitial pattern throughout the lungs that silhouettes pulmonary blood vessels. accompanied by increase in number of chronic inflammatory cells mixed with bronchial epithelial cells in TBA smears, suggestive of lung pathology. Thus, bronchial pattern could not be overlooked and was considered a part of disease complex.

\section{Acute pneumonia}

Radiographic findings were widely variable in acute pneumonia. Unstructured interstitial pattern and pleural effusions were observed in equal proportions (33.3\%). Mixed lung pattern was recorded in three cows. In pleural effusions, pulmonary parenchymal lesions were not discernable, however TBA cytology was more diagnostic in acute pneumonia than radiography. Pleural effusions were more likely to be of primary origin because of spread of infection from lungs to pleural space to cause pleuropneumonia. Unstructured interstitial pattern in acute pneumonia in six cows was attributed to pneumonitis, viral diseases and bronchopneumonia. Interstitial pattern was reported in three of nine cows suffering from bronchopneumonia (Masseau et al., 2008).

\section{Aspiration pneumonia}

Chest radiography revealed nodular interstitial pattern (50\%) as the most common radiographic sign, followed by unstructured interstitial pattern and pleural effusions. There was no radiographic sign in two cattle. No characterstic radiographic lesions in aspiration pneumonia were attributed to involvement of cranial lung lobe in aspiration pneumonia and accessibility of only caudal lung lobe in radiography.

\section{Tuberculosis}

It was characterized by nodular interstitial pattern (Fig 7) in three of five cattle and miliary interstitial pattern in other two (Fig 8). These patterns were attributed to chronic granulomatous lesions characteristic in mycobacterial infectons. These lung patterns were also observed in chronic pneumonia. Miliary lesions on chest radiography were also observed in tuberculosis affected cattle (Buakgava and Khanna 1987). In humans as well, miliary lesions in both lungs were reported in pulmonary form of tuberculosis (Hamid et al., 1991).

\section{Survivability in diseased cattle}

Maximum survivability was recorded in cattle with bronchial $(81.8 \%)$ followed by unstructured interstitial $(57.1 \%)$ and nodular interstitial pattern (53.3\%). Survivability was least in cattle manifesting miliary interstitial pattern where all the three animals died. One out of two diseased cattle with normal lung pattern also died due to severe aspiration pneumonia. Survivability was maximum in chronic pneumonia (70.8 per cent) followed by aspiration pneumonia $(62.5 \%)$ and acute pneumonia (44.4\%). None of the cattle with tuberculosis survived (Table 1).

Besides the type of lung pattern, the severity of lung pattern was also correlated with the survivability (Table 2). Generally survivability decreased with severity of lung pattern. Equal proportion of survivability (4/6) was observed 
in moderate and marked interstitial pattern, but none survived $(0 / 2)$ with mild interstitial pattern, which could be due to systemic infection or focal lesions in lung lobes not accessible to radiography. Survivability was high in mild and moderate nodular pattern than marked nodular pattern. Survivability was $3 / 3$ in mild and $5 / 7$ in moderate bronchial pattern. Survivability decreased with severity of lung pattern in general, as expected. Similar to present study, more favorable prognosis was reported in chronic interstitial pneumonia than acute interstitial pneumonia (Nout et al. 2002).

\section{CONCLUSION}

Based on the present study, correlation between radiographic signs and tracheo-bronchial aspirate (TBA) cytology and clinical findings can aid in accurate diagnosis and prognosis of pneumonia in cattle.

\section{REFERENCES}

Buakgava, A.K. and Khanna, P.N. (1987). Tuberculosis in animalsA zoonotic problem. Indian Journal of Tuberculosis. 34: 200.

Elsiddig, I.A.M. (2003). Pathological changes in the lungs of slaughtered sheep and cattle in Khartoum state abattoirs. M.V.Sc Thesis, Department of Pathology, Faculty of Veterinary Medicine, University of Khartoum.

Gabinaitiene, A., Siugzdaite, J., Zilinskas, H., Siugzda, R. and Petkevicius, S. (2011). Mycoplasma bovis and bacterial pathogens in the bovine respiratory tract. Veterinarni Medicina. 56(1): 28-34.

Hamid, M.E., Mohammed, G.E., Abu-Samra, M.T., Elsanousi, S.M. and Barri, M.E. (1991). Bovine farcy: A clinico-pathological study of the disease and its aetiological agent. Journal of Comparative Pathology. 105: 287-301.
Lekeux P. (1988). Spécificité de la fonctionpulmonaire des jeunes bovines. Proc Maladies respiratoires des jeunes bovines. Congres SFB. Ed J. Espinasse. pp. 108-05.

Masseau, I., Fecteau, G., Breton, L., Hélie, P., Beauregard, G. and Blond, L. (2008). Radiographic detection of thoracic lesions in adult cows: A retrospective study of 42 cases (19952002). Can Vet J. 49: 261-267.

Meena, B.K. (2012). Radiological diagnosis of thoraco-abdominal disorders in large ruminants. M.V.Sc. Thesis. Rajasthan University of Veterinary and Animal Sciences, Bikaner, India.

Nout, Y.S., Hinchcliff, K.W., Samii, V.F., Kohn, C.W., Jose-Cunilleras, E. and Reed, S.M. (2002). Chronic pulmonary disease with radiographic interstitial opacity (interstitial pneumonia) in foals. Equine Veterinary Journal. 34(6): 542-48.

Nykamp, S.G. (2013). The Equine Thorax In: Textbook of Veterinary Diagnostic Radiology. [Thrall, D.E. (Ed.)] $6^{\text {th }}$ Edn. Elsevier pp. 632-646.

Sorden, S.D., Kerr. R.W. and Janzen, E.D. (2000). Interstitial pneumonia in feedlot cattle: concurrent lesions and lack of immunohistochemical evidence for bovine respiratory syncytial virus infection. Journal of Veterinary Diagnostic Investigation. 12(6): 510-517.

Tanwar, M. (2016). Radiological Diagnosis Using Computed Radiography for Thoraco-abdominal Disorders of Bovines. Ph.D. Thesis. Rajasthan University of Veterinary and Animal Sciences, Bikaner, India.

Verschooten, F., Oyaert, W. and Drubbel, R. (1974). Radiographic diagnosis of lung diseases in cattle. Journal of American Veterinary Radiology Societies. 15: 49-59.

Wilkins, P.A. and Woolums, A.R. (2009). Diseases of the Respiratory System In: [Smith, B.P. (Ed.)] Large Animal Internal Medicine. $4^{\text {th }}$ ed. California: Mosby, Elsevier. pp. 490-666. 